\title{
Journey to the Center of the Linear Ordering Problem
}

Leticia Hernando

leticia.hernando@ehu.eus

University of the Basque Country UPV/EHU, 48940 Leioa, Spain

\author{
Alexander Mendiburu \\ alexander.mendiburu@ehu.eus \\ University of the Basque Country UPV/EHU, 20018 San Sebastián, Spain
}

Jose A. Lozano

ja.lozano@ehu.eus

Basque Center for Applied Mathematics (BCAM), 48009 Bilbao, Spain

University of the Basque Country UPV/EHU, 20018 San Sebastián, Spain

\begin{abstract}
A number of local search based algorithms have been designed to escape from the local optima, such as, iterated local search or variable neighborhood search. The neighborhood chosen for the local search as well as the escape technique play a key role in the performance of these algorithms. Of course, a specific strategy has a different effect on distinct problems or instances. In this paper, we focus on a permutation-based combinatorial optimization problem: the linear ordering problem. We provide a theoretical landscape analysis for the adjacent swap, the swap and the insert neighborhoods. By making connections to other different problems found in the Combinatorics field, we prove that there are some moves in the local optima that will necessarily return a worse or equal solution. The number of these non-better solutions that could be avoided by the escape techniques is considerably large with respect to the number of neighbors. This is a valuable information that can be included in any of those algorithms designed to escape from the local optima, increasing their efficiency.
\end{abstract}

\section{Keywords}

Permutation-based Combinatorial Optimization Problems, Linear Ordering Problem, Local Optima, Landscape Analysis

\section{Introduction}

Local search based algorithms have been used to solve permutation-based combinatorial optimization problems. The steps followed by these algorithms are defined according to a structure of the search space which is given by the neighborhood. The concept of neighborhood together with the combinatorial optimization problem is known as the landscape (Reidys and Stadler, 2002). The neighborhood plays a key role in the performance of the local search based algorithms. Usually, the algorithm stops when a local optimum is reached, that is, a permutation (solution) whose neighbors are not better than itself. Different strategies have been developed trying to escape from the local optima where the local search is trapped. In this connection, a number of algorithms, such as iterated local search or variable 
neighborhood search, have appeared in the literature (Lourenço et al., 2010, 2003; Stützle, 2006; Marmion et al., 2011; Mladenović and Hansen, 1997; Hansen and Mladenović, 2018; Schiavinotto and Stützle, 2005).

Some of these proposals are metaheuristic algorithms, which have a general design and which do not take into account the specific properties of the given problem (Lourenço et al., 2010, 2003; Mladenović and Hansen, 1997; Garcia et al., 2006). While other heuristic techniques have been developed for particular permutation-based combinatorial optimization problems (Stützle, 2006; Schiavinotto and Stützle, 2005; Santucci and Ceberio, 2020). In the last few decades, researchers have seen the necessity to carry out landscape analyses more than to continue to propose new algorithms without knowledge about the problem at hand (Hernando et al., 2019a, 2018; Albrecht et al., 2008; Alyahya and Rowe, 2014; Chicano et al., 2012; Humeau et al., 2013; Schiavinotto and Stützle, 2003).

In particular, the study of the linear ordering problem has been the focus of numerous articles and books (Marti and Reinelt, 2011; Schiavinotto and Stützle, 2005, 2003). Given a matrix of numerical values, the linear ordering problem consists of finding a simultaneous permutation of the rows and columns, such that the sum of the entries above the main diagonal is maximized. This problem has attracted the attention of researchers because it has very special properties that make it apparently really easy to solve; however, it is an NP-hard problem (Mishra and Sikdar, 2004). For example, the symmetry enclosing this problem should be highlighted (Marti and Reinelt, 2011; Hernando et al., 2019b): the solution that maximizes the objective function is the reverse of the one that minimizes it, or in general, the reverse of the $k$-th best solution is the $k$-th worst solution. Moreover, in a recent contribution (Ceberio et al., 2015), it was found that the global optimum could not contain some items in specific positions of the permutation. As can be seen, the linear ordering problem is latent in the literature and, although great progress has been made, there are still many hidden details.

In this paper, we present a landscape analysis for the linear ordering problem. We focus on the study of the objective function values of certain solutions when a local optimum is reached under different neighborhoods: the adjacent swap, the swap and the insert. The fact that a local optimum is found implies that a number of restrictions must be fulfilled in the input matrix of the instance. These restrictions are based on the $\Delta$-function evaluation for the linear ordering problem (Schiavinotto and Stützle, 2005). We theoretically prove that they cause considerably large number of solutions to be necessarily worse than or equal to the local optimum. We generalize this issue for all permutations of size $n$ and show how to build those permutations that do not belong to this set of non-better solutions. This generalization is made thanks to the association of each of our cases, according to the neighborhood, with different known problems in the Combinatorics field, never done before. The utility of this work is clear, as our theoretical analysis provides valuable information so as to be able to escape from the local optima under the three most commonly used neighborhoods.

The rest of the paper is organized as follows. The linear ordering problem, as well as the neighborhoods used in the analysis, are formally introduced in Section 2. In Section 3, we carry out the theoretical study about the implications that arise when a local optima is found under the different neighborhoods, and the relevance of these new findings is discussed in Section 4. Finally, in Section 5, the conclusions and future work are presented. 


\section{Preliminary concepts}

\subsection{Linear Ordering Problem}

The linear ordering problem (LOP) is a permutation-based combinatorial optimization problem that commonly appears in economics when analyzing the interrelations among the sectors of an economy, or in the study of relationships among countries. Given a matrix $A=\left[a_{i j}\right]_{n \times n}$ of numerical values, the LOP consists of finding a simultaneous permutation $\sigma \in S_{n}$ of the rows and columns of $A$, such that the sum of the entries above the main diagonal is maximized. The solutions are coded as permutations $\sigma=(\sigma(1) \sigma(2) \cdots \sigma(n-1) \sigma(n))$, where $\sigma(i)$ represents the number of the row (and column) of $A$ that is located at the $i$-th position. So, the search space $S_{n}$ is the whole set of permutations of size $n$, and thus its size is $\left|S_{n}\right|=n$ !. Formally, the aim is to find a permutation $\sigma$ in such a way that the following function is maximized:

$$
f(\sigma)=\sum_{i=1}^{n-1} \sum_{j=i+1}^{n} a_{\sigma(i) \sigma(j)} .
$$

\subsection{Neighborhoods}

A landscape is defined as the triple $\left(f, S_{n}, \mathcal{N}\right)$ where $f$ is the objective function to optimize, $S_{n}$ is the search space and $\mathcal{N}$ is the neighborhood (Reidys and Stadler, 2002). The most commonly used neighborhoods for permutations are the adjacent swap, the swap (or 2-exchange) and the insert neighborhoods (Schiavinotto and Stützle, 2005).

\subsubsection{Adjacent swap}

Given $\sigma=(\sigma(1) \sigma(2) \cdots \sigma(n)) \in S_{n}$, its adjacent swap neighboring solutions are those permutations obtained as the result of swapping two adjacent elements of such solution $\sigma$. For example, in the space of permutations of size $n=4$, the set of adjacent swap neighbors of the permutation $\sigma=(1234)$ is: $\mathcal{N}_{A}(1234)=\{(2134),(1324),(1243)\}$. The number of adjacent swap neighbors of a permutation of size $n$ is $n-1$.

\subsubsection{Swap or 2-exchange}

The swap or 2-exchange neighborhood considers that two solutions are neighbors if one is generated by swapping two elements of the other, not necessarily adjacent. Taking the same permutation $\sigma=(1234)$ as in the previous example, the set composed of its neighbors under the swap neighborhood is: $\mathcal{N}_{S}(1234)=\{(2134),(3214),(4231),(1324),(1432),(1243)\}$. Under this neighborhood, a permutation has $n(n-1) / 2$ neighbors.

\subsubsection{Insert}

A neighboring permutation under this neighborhood is obtained by moving an element to a different position. Following the same example, $\mathcal{N}_{I}(1234)=$ $\{(2134),(2314),(2341),(1324),(1342),(3124),(1243),(4123),(1423)\}$. The number of neighbors in this case is $(n-1)^{2}$. 


\section{Discarding solutions of the search space}

Assuming a maximization problem, a local optimum under a neighborhood $\mathcal{N}$ is defined as a solution $\sigma^{*}$ such that $f\left(\sigma^{*}\right) \geq f(\sigma), \forall \sigma \in \mathcal{N}\left(\sigma^{*}\right)$. So, the permutations that are local optima under the adjacent swap, the swap and the insert neighborhoods are, at least, better than or equal to $n-1, n(n-1) / 2$ and $(n-1)^{2}$ solutions, respectively. However, for the linear ordering problem, more information can be collected from a local optimum $\sigma^{*}$ : apart from those neighboring solutions, there is a set of permutations of the search space that will never be better than the local optimum. The $\Delta$-function evaluations for the linear ordering problem (Schiavinotto and Stützle, 2005) are the basis for the analysis developed in this work.

\subsection{Analysis for the Adjacent Swap}

Example. If $\sigma^{*}=(1234) \in S_{4}$ is a local optimum under the adjacent swap neighborhood, then, by definition $f\left(\sigma^{*}\right) \geq f(\sigma), \forall \sigma \in \mathcal{N}_{A}\left(\sigma^{*}\right)$. Considering the three neighbors of $\sigma^{*}$ detailed in Section 2.2.1, the following three inequalities are obtained:

- For $\sigma_{1}=(2134): f(1234) \geq f(2134) \Rightarrow$

$$
\begin{gathered}
\Rightarrow a_{12}+a_{13}+a_{14}+a_{23}+a_{24}+a_{34} \geq a_{21}+a_{23}+a_{24}+a_{13}+a_{14}+a_{34} \\
\Rightarrow a_{12} \geq a_{21}
\end{gathered}
$$

- For $\sigma_{2}=(1324): f(1234) \geq f(1324) \Rightarrow$

$$
\begin{gathered}
\Rightarrow a_{12}+a_{13}+a_{14}+a_{23}+a_{24}+a_{34} \geq a_{13}+a_{12}+a_{14}+a_{32}+a_{34}+a_{24} \\
\Rightarrow a_{23} \geq a_{32}
\end{gathered}
$$

- For $\sigma_{3}=(1243): f(1234) \geq f(1243) \Rightarrow$

$$
\begin{gathered}
\Rightarrow a_{12}+a_{13}+a_{14}+a_{23}+a_{24}+a_{34} \geq a_{12}+a_{14}+a_{13}+a_{24}+a_{23}+a_{43} \\
\Rightarrow a_{34} \geq a_{43}
\end{gathered}
$$

The result shown in this example is generalized in Theorem 1, where the necessary and sufficient conditions for a solution to be a local optimum under the adjacent swap neighborhood are given for any permutation size $n$.

Theorem 1. A solution $\sigma^{*}=\left(\sigma^{*}(1) \sigma^{*}(2) \cdots \sigma^{*}(n)\right) \in S_{n}$ is a local optimum for the LOP under the adjacent swap neighborhood if and only if the input matrix $A=\left[a_{i j}\right]_{n \times n}$ of the LOP instance fulfills:

$$
a_{\sigma^{*}(i) \sigma^{*}(i+1)} \geq a_{\sigma^{*}(i+1) \sigma^{*}(i)}, \forall i=1, \ldots, n-1 .
$$

Proof. First, let us suppose that a permutation $\sigma^{*}=\left(\sigma^{*}(1) \sigma^{*}(2) \cdots \sigma^{*}(n)\right) \in S_{n}$ is a local optimum for the LOP under the adjacent swap neighborhood. The objective function of $\sigma^{*}$ is:

$$
f\left(\sigma^{*}\right)=\sum_{k=1}^{n-1} \sum_{j=k+1}^{n} a_{\sigma^{*}(k) \sigma^{*}(j)} .
$$


The neighbors of $\sigma^{*}$ are the permutations $\sigma_{i}$, such that

$$
\sigma_{i}=\left(\sigma^{*}(1) \cdots \sigma^{*}(i+1) \sigma^{*}(i) \cdots \sigma^{*}(n)\right), \quad i=1, \ldots, n-1,
$$

that is, $\sigma_{i}(k)=\sigma^{*}(k), \forall k \neq i, i+1$, and $\sigma_{i}(i)=\sigma^{*}(i+1)$ and $\sigma_{i}(i+1)=\sigma^{*}(i)$. Thus, their objective function values are:

$$
f\left(\sigma_{i}\right)=f\left(\sigma^{*}\right)-a_{\sigma^{*}(i) \sigma^{*}(i+1)}+a_{\sigma^{*}(i+1) \sigma^{*}(i)}, \quad \forall i=1, \ldots, n-1 .
$$

As $\sigma^{*}$ is a local optimum, $f\left(\sigma^{*}\right) \geq f\left(\sigma_{i}\right), \forall \sigma_{i} \in \mathcal{N}_{A}\left(\sigma^{*}\right)$, and therefore, the inequalities in (1) must be fulfilled.

Second, as has been argued before, when comparing $f\left(\sigma^{*}\right)$ with $f\left(\sigma_{i}\right), \forall \sigma^{*} \in S_{n}$, $\forall \sigma_{i} \in \mathcal{N}_{A}\left(\sigma^{*}\right): f\left(\sigma_{i}\right)=f\left(\sigma^{*}\right)-a_{\sigma^{*}(i) \sigma^{*}(i+1)}+a_{\sigma^{*}(i+1) \sigma^{*}(i)}$. If the inequalities in (1) are fulfilled for $\sigma^{*}$, then:

$$
f\left(\sigma_{i}\right)=f\left(\sigma^{*}\right)-a_{\sigma^{*}(i) \sigma^{*}(i+1)}+a_{\sigma^{*}(i+1) \sigma^{*}(i)} \leq f\left(\sigma^{*}\right),
$$

$\forall \sigma_{i} \in \mathcal{N}_{A}\left(\sigma^{*}\right)$. Thus, $\sigma^{*}$ is, indeed, a local optimum.

Theorem 1 provides valuable knowledge about the objective function value of more solutions of the search space, apart from the neighboring solutions. That is, there is a set of permutations that are not neighbors of the local optimum, but such that, when comparing their objective function values with that of the local optimum, a number of restrictions (at least two) in (1) can be considered so as to conclude that they are also worse than or equal to the local optimum. For example, let's consider that the permutation $\sigma^{*}=(123456) \in S_{6}$ is a local optimum. In Figure 1 top-left, the matrix solution represented by this identity permutation is shown, where the elements above the main diagonal, which are those involved in the objective function, are shadowed. Swapping items $1-2$ from $\sigma^{*}$, the solution codified by the permutation $(213456) \in \mathcal{N}_{A}\left(\sigma^{*}\right)$ is obtained (Figure 1 top-right). Comparing the objective function value of this solution with that of the local optimum, we observe that the element $a_{12}$ (in red) is subtracted while $a_{21}$ (in blue) is added. Thus, the element in red must be greater than or equal to the element in blue: $a_{12} \geq a_{21}$. The solution $(123546) \in \mathcal{N}_{A}\left(\sigma^{*}\right)$, obtained from swapping items $4-5$ from $\sigma^{*}$, is given in Figure 1 bottom-left. This time it is observed that the element $a_{45}$ (in red) is subtracted while $a_{54}$ (in blue) is added when comparing with the objective function of $\sigma^{*}$, thus, $a_{45} \geq a_{54}$. Finally, the permutation $\sigma=(213546) \notin \mathcal{N}_{A}\left(\sigma^{*}\right)$ is the result of swapping the elements $1-2$ and $4-5$ of $\sigma^{*}$ and represents the matrix given in Figure 1 bottom-right. This solution fulfils that $f\left(\sigma^{*}\right) \geq f(\sigma)$, because $a_{12} \geq a_{21}$ and $a_{45} \geq a_{54}$. However, we can not prove that, for example, the solution $\sigma=(231456) \notin \mathcal{N}_{A}\left(\sigma^{*}\right)$, which is the result of swapping the elements $1-2$ and then $1-3$ of $\sigma^{*}$, is not better than $\sigma^{*}$. When comparing their objective function values, we find they share all terms except $a_{12}+a_{13}$ and $a_{21}+a_{31}$. Because of eq (1), $a_{12} \geq a_{21}$, but there is not a known relation between $a_{13}$ and $a_{31}$. Thus, in this case, we can not prove that $f\left(\sigma^{*}\right) \geq f(\sigma)$.

Precisely, as can be seen in the example, if a solution is the result of swapping two or more pairs of adjacent items of the local optimum in such a way that the adjacent swaps do not intersect with each other, then, the solution will never be better than the local optimum. The question that arises is: how many solutions of this form can be 


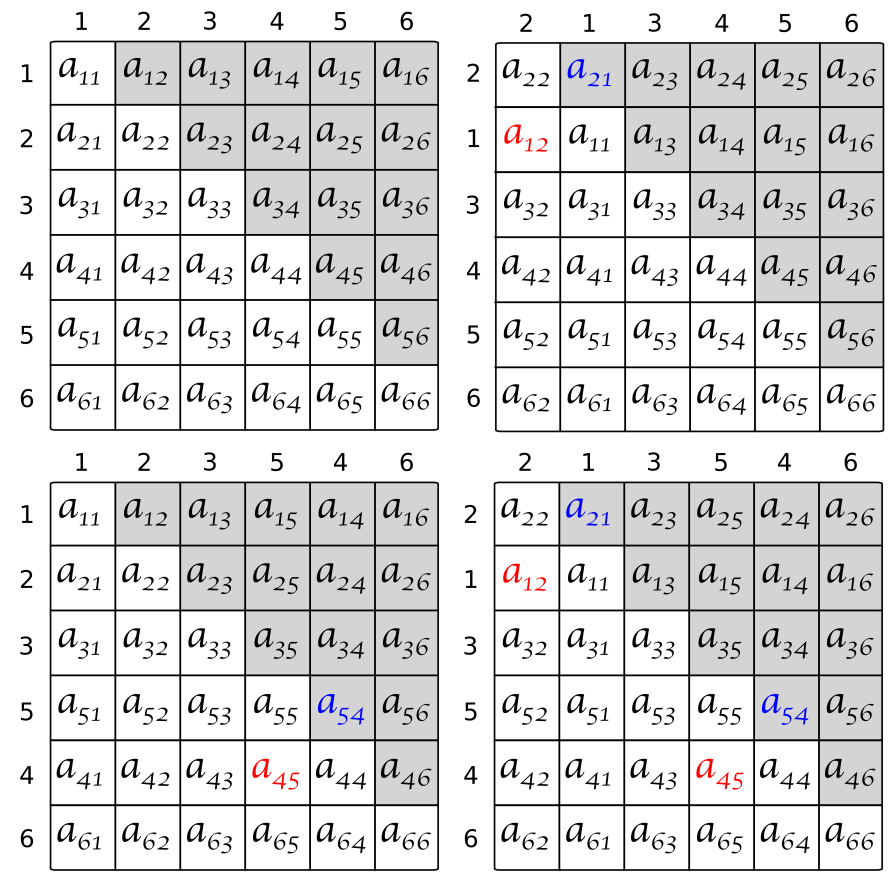

Figure 1: Matrix solutions of the LOP represented by the permutations (123456), (213456), (123546) and (213546). The elements that are involved in the objective function are shadowed. Comparing the objective function value of each solution with that of the local optimum, the elements subtracted are in red while the elements added are in blue.

built? In order to answer to this question, we may refer to the Fibonacci Numbers. The Fibonacci sequence is extensively known in the Combinatorics field (Scott and Marketos, 2014). The natural definition of the numbers $\mathcal{F}_{i}$ that form this sequence is given as follows:

$$
\mathcal{F}_{1}=1, \mathcal{F}_{2}=1, \mathcal{F}_{n}=\mathcal{F}_{n-1}+\mathcal{F}_{n-2}, \forall n \geq 3 .
$$

However, the Fibonacci Numbers can also be defined as:

$$
\mathcal{F}_{n+1}=\sum_{i=0}^{\left\lfloor\frac{n}{2}\right\rfloor}\left(\begin{array}{c}
n-i \\
i
\end{array}\right), \forall n \geq 0,
$$

which is the total number of ways of choosing adjacent pairs from $n$ elements, where the same element is not chosen in more than one pair. So, it counts the number of permutations of size $n$ that are the result of swapping two or more pairs of adjacent items in such a way that the adjacent swaps do not intersect with each other.

In conclusion, finding a local optimum $\sigma^{*} \in S_{n}$ for the LOP under the adjacent swap neighborhood implies that $k_{n}^{A}$ solutions are not better than $\sigma^{*}$, where $k_{n}^{A}=\mathcal{F}_{n+1}$ is the $(n+1)$-th Fibonacci Number. Moreover, we know how to build the solutions that could be better than the local optimum: by applying a number of sequent adjacent swaps to the items of the local optimum, where at least one item is involved in more 
than one adjacent swap. We can not guarantee the resultant solution to be better than the local optimum, but we do know that by applying non-intersecting adjacent swaps to $\sigma^{*}$ no better solutions are reached.

\subsection{Analysis for the Swap}

Example. If $\sigma^{*}=(1234) \in S_{4}$ is a local optimum under the swap neighborhood, then $f\left(\sigma^{*}\right) \geq f(\sigma), \forall \sigma \in \mathcal{N}_{S}\left(\sigma^{*}\right)$. As in Example 3.1, a number of inequalities are obtained just by comparing the objective function value of the local optimum $\sigma^{*}$ with the value of each of the neighboring solutions:

$$
\begin{aligned}
a_{12} & \geq a_{21} \\
a_{12}+a_{13}+a_{23} & \geq a_{21}+a_{31}+a_{32} \\
a_{12}+a_{13}+a_{14}+a_{24}+a_{34} & \geq a_{21}+a_{31}+a_{41}+a_{42}+a_{43} \\
a_{23} & \geq a_{32} \\
a_{23}+a_{24}+a_{34} & \geq a_{32}+a_{42}+a_{43} \\
a_{34} & \geq a_{43}
\end{aligned}
$$

This result is generalized in Theorem 2 for any permutation size.

Theorem 2. A solution $\sigma^{*}=\left(\sigma^{*}(1) \sigma^{*}(2) \cdots \sigma^{*}(n)\right) \in S_{n}$ is a local optimum for the LOP under the swap neighborhood if and only if the input matrix $A=\left[a_{i j}\right]_{n \times n}$ of the LOP instance fulfills:

$$
\begin{gathered}
\sum_{k=i+1}^{j} a_{\sigma^{*}(i) \sigma^{*}(k)}+\sum_{k=i}^{j-1} a_{\sigma^{*}(k) \sigma^{*}(j)} \geq \\
\geq \sum_{k=i+1}^{j} a_{\sigma^{*}(k) \sigma^{*}(i)}+\sum_{k=i}^{j-1} a_{\sigma^{*}(j) \sigma^{*}(k)}, \\
\forall i=1,2, \ldots, n-1, \quad \forall j=i+1, \ldots, n .
\end{gathered}
$$

Proof. First, let us suppose that a permutation $\sigma^{*}=\left(\sigma^{*}(1) \sigma^{*}(2) \cdots \sigma^{*}(n)\right) \in S_{n}$ is a local optimum for the LOP under the swap neighborhood. We denote the neighbors of $\sigma^{*}$ by $\sigma_{i j}$, where $\forall i=1 \ldots, n-1, \forall j=i+1, \ldots, n, \sigma_{i j}=\left(\sigma^{*}(1) \cdots \sigma^{*}(i-1) \sigma^{*}(j) \sigma^{*}(i+\right.$ 1) $\left.\cdots \sigma^{*}(j-1) \sigma^{*}(i) \sigma^{*}(j+1) \cdots \sigma^{*}(n)\right)$. Thus, their objective function values are $\forall i=$ $1, \ldots, n-1, \forall j=i+1, \ldots, n$ :

$$
\begin{aligned}
f\left(\sigma_{i j}\right)= & f\left(\sigma^{*}\right)-\sum_{k=i+1}^{j} a_{\sigma^{*}(i) \sigma^{*}(k)}-\sum_{k=i}^{j-1} a_{\sigma^{*}(k) \sigma^{*}(j)}+ \\
& +\sum_{k=i+1}^{j} a_{\sigma^{*}(k) \sigma^{*}(i)}+\sum_{k=i}^{j-1} a_{\sigma^{*}(j) \sigma^{*}(k)} .
\end{aligned}
$$

As $\sigma^{*}$ is a local optimum, $f\left(\sigma^{*}\right) \geq f\left(\sigma_{i j}\right), \forall \sigma_{i j} \in \mathcal{N}_{S}\left(\sigma^{*}\right)$ and therefore, the inequalities in (2) must be fulfilled. 
Second, as has been argued before, when comparing $f\left(\sigma^{*}\right)$ with $f\left(\sigma_{i j}\right), \forall \sigma^{*} \in$ $S_{n}, \forall \sigma_{i j} \in \mathcal{N}_{S}\left(\sigma^{*}\right): \quad f\left(\sigma_{i j}\right)=f\left(\sigma^{*}\right)-\sum_{k=i+1}^{j} a_{\sigma^{*}(i) \sigma^{*}(k)}-\sum_{k=i}^{j-1} a_{\sigma^{*}(k) \sigma^{*}(j)}+$ $\sum_{k=i+1}^{j} a_{\sigma^{*}(k) \sigma^{*}(i)}+\sum_{k=i}^{j-1} a_{\sigma^{*}(j) \sigma^{*}(k)}$. If the inequalities in (2) are fulfilled for $\sigma^{*}$, then $f\left(\sigma_{i j}\right) \leq f\left(\sigma^{*}\right), \forall \sigma_{i j} \in \mathcal{N}_{S}\left(\sigma^{*}\right)$, and thus, $\sigma^{*}$ is a local optimum.

\begin{tabular}{|c|c|c|c|c|c|c|c|c|c|c|c|c|c|}
\hline & & & & & $J$ & & & & & & & & \\
\hline & $a_{11}$ & $a_{12}$ & $a_{13}$ & $a_{14}$ & $a_{15}$ & 16 & & $a_{33}$ & $a_{32}$ & $a_{31}$ & $a_{34}$ & $a_{35}$ & 36 \\
\hline & $a_{21}$ & 22 & & 24 & $a_{25}$ & & & $l_{23}$ & $a_{22}$ & $a_{21}$ & 24 & $a_{25}$ & 126 \\
\hline & $a$ & 32 & & 34 & $a_{35}$ & 36 & & $l_{13}$ & 12 & $a_{11}$ & $l_{14}$ & $a_{15}$ & 16 \\
\hline & +1 & 42 & & 44 & 45 & 46 & & $l_{43}$ & $a_{42}$ & $a_{41}$ & 44 & $a_{45}$ & 4 \\
\hline & $a_{51}$ & 52 & $a_{53}$ & 54 & $a_{55}$ & 56 & & $a_{53}$ & $a_{52}$ & $a_{51}$ & $l_{54}$ & $a_{55}$ & $l_{56}$ \\
\hline & $a$ & $a_{62}$ & $a_{63}$ & 64 & $a$ & 66 & & $a_{63}$ & $a_{62}$ & $a_{61}$ & $a_{64}$ & $a_{65}$ & $\lambda_{6}$ \\
\hline & 1 & & & & & & & 3 & & & 6 & 5 & 4 \\
\hline & $a_{11}$ & 2 & $a_{13}$ & 6 & $a_{15}$ & 14 & & $a_{33}$ & $a_{32}$ & $a_{31}$ & $a_{36}$ & $a_{35}$ & $a_{34}$ \\
\hline & $a_{21}$ & 22 & $a_{23}$ & & 25 & 24 & & $a_{23}$ & $a_{22}$ & $a_{21}$ & 26 & $a_{25}$ & 24 \\
\hline & $a_{3}$ & & c & 6 & $a_{35}$ & 4 & & 13 & 12 & $a_{11}$ & $a_{16}$ & $a_{15}$ & $l_{1}$ \\
\hline & $a_{61}$ & 62 & $a_{63}$ & $a_{66}$ & 65 & 64 & & $a_{63}$ & $a_{62}$ & $a_{61}$ & $a_{66}$ & $a_{65}$ & 62 \\
\hline & $a_{51}$ & 2 & $a_{53}$ & 6 & $a_{55}$ & 54 & & $a_{53}$ & $a_{52}$ & $a_{51}$ & $a_{56}$ & $a_{55}$ & $a_{5}$ \\
\hline & & & $a_{43}$ & & 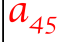 & & & $a_{43}$ & $a_{42}$ & $a_{41}$ & & $a_{45}$ & 4 \\
\hline
\end{tabular}

Figure 2: Matrix solutions of the LOP represented by the permutations (123456), (321456), (123654) and (321654). The elements that are involved in the objective function are shadowed, and comparing the objective function value of each solution with that of the local optimum, the elements subtracted are in red while the elements added are in blue.

Theorem 2 provides knowledge about the objective function value of more solutions of the search space, apart from the neighboring solutions. Again, there are non-neighboring permutations of the local optimum, such that, when comparing their objective function values with that of the local optimum, a number of restrictions in (2) can be considered so as to conclude that they are not better than the local optimum. Following with the previous example, let's consider that the permutation $\sigma^{*}=(123456) \in S_{6}$ is a local optimum. In Figure 2 top-left, the matrix solution represented by this permutation is shown. Swapping items $1-3$ from $\sigma^{*}$, the solution codified by the permutation $(321456) \in \mathcal{N}_{S}\left(\sigma^{*}\right)$ is obtained (Figure 2 top-right). When the objective function value of this solution is compared with that of the local optimum, the elements $a_{12}, a_{13}$ and $a_{23}$ (in red) are subtracted while $a_{21}, a_{31}$ and $a_{32}$ (in blue) are added. Thus, the sum of the elements in red must be greater than or equal to the sum of the elements in blue: $a_{12}+a_{13}+a_{23} \geq a_{21}+a_{31}+a_{32}$. The solution 
$(123654) \in \mathcal{N}_{S}\left(\sigma^{*}\right)$, obtained from swapping items $4-6$ from $\sigma^{*}$, is given in Figure 2 bottom-left. The elements $a_{45}, a_{46}$ and $a_{56}$ (in red) are subtracted while $a_{54}, a_{64}$ and $a_{65}$ (in blue) are added when comparing with the objective function of $\sigma^{*}$, thus, $a_{45}+a_{46}+a_{56} \geq a_{54}+a_{64}+a_{65}$. The permutation $\sigma=(321654) \notin \mathcal{N}_{S}\left(\sigma^{*}\right)$ is the result of swapping the elements $1-3$ and $4-6$ of $\sigma^{*}$ and represents the matrix given in Figure 2 bottom-right. This solution fulfils that $f\left(\sigma^{*}\right) \geq f(\sigma)$. Nevertheless, taking, for instance, the solution $\sigma=(453126) \notin \mathcal{N}_{S}\left(\sigma^{*}\right)$, which is the result of swapping the elements $1-4$ and $2-5$ of $\sigma^{*}$, we can not prove that $f\left(\sigma^{*}\right) \geq f(\sigma)$ just with restrictions in (2).

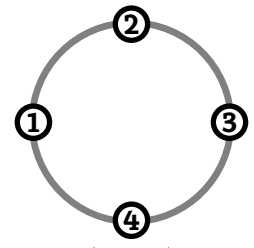

(1234)

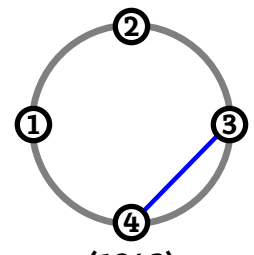

(1243)

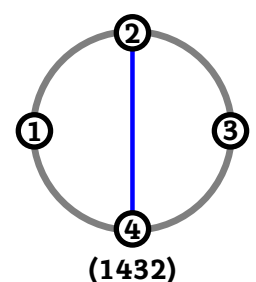

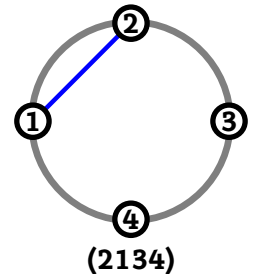

(2134)

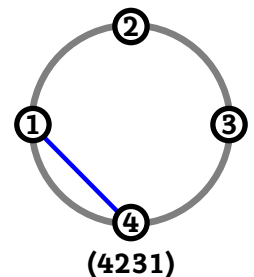

(4231)

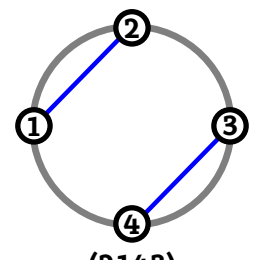

(2143)

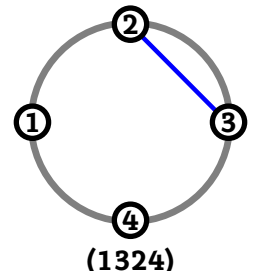

(1324)
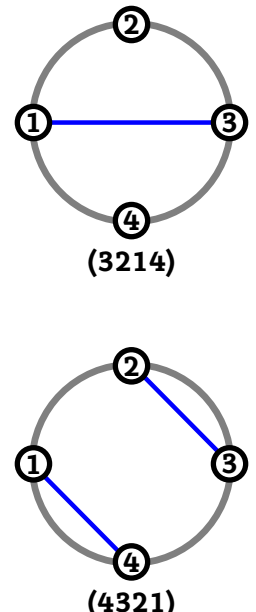

Figure 3: Representation of the Motzkin Numbers $\mathcal{M}_{n}$ for $n=4$ as points located in a circle drawing non-intersecting chords (in blue) between pairs of points. Association between them and the permutations of size 4 derived from applying non-crossing swaps to the items of the identity permutation. The $1^{\text {st }}$ circle represents the identity permutation $\sigma^{*}=(1234) ; 2^{n d}$ to $7^{t h}$ circles indicate the neighbors of $\sigma^{*}$ with just one chord; $8^{t h}$ and $9^{\text {th }}$ circles refer to solutions at distance 2 from $\sigma^{*}$, with two chords.

As can be seen in the example, if a solution is the result of swapping two or more pairs of items of the local optimum in such a way that the swaps do not cross nor intersect with each other, then, the solution will never be better than the local optimum. In order to generalize this aspect and to provide a general rule for all $n$ with the aim of finding this kind of solutions, we may refer to the Motzkin Numbers (Aigner, 1998). For a given number $n$, the Motzkin Number $\mathcal{M}_{n}$ is the number of different ways of drawing non-intersecting chords between pairs of $n$ points on a circle (not necessarily touching every point by a chord). Transferring this insight to our 
problem, we propose considering that the points represent the items $\sigma(1), \ldots, \sigma(n)$ of the permutation, and that each chord that goes from $\sigma(i)$ to $\sigma(j)$ indicates that items $\sigma(i)$ and $\sigma(j)$ are swapped. In Figure 3, this association between the Motzkin Numbers and the swaps in the permutations is represented for $n=4$ points. All the possibilities of connecting pairs of points by non-intersecting chords, or equivalently, all the possibilities of swapping items of $\sigma^{*}=(1234)$ without crossing swaps are considered. Figure 3 shows that for $n=4$ there is a total of 9 solutions that are not generated by applying intersecting swaps: the input permutation that represents the local optimum (1234), the 6 neighboring solutions represented just with one chord (2134), (1324), (1243), (4231), (3214), (1432), and 2 solutions at distance two from the local optimum represented with two chords (2143), (4321).

The Motzkin Numbers are defined by the recurrence relation:

$$
\mathcal{M}_{n+1}=\mathcal{M}_{n}+\sum_{i=0}^{n-1} \mathcal{M}_{i} \mathcal{M}_{n-1-i}, \forall n \geq 1,
$$

with $\mathcal{M}_{0}=1$ and $\mathcal{M}_{1}=1$. They can also be expressed in terms of Catalan numbers $\mathcal{C}_{k}$ (Riordan, 1968):

$$
\mathcal{M}_{n}=\sum_{k=0}^{\lfloor n / 2\rfloor}\left(\begin{array}{c}
n \\
2 k
\end{array}\right) \mathcal{C}_{k}=\frac{(2 n) !}{(n+1) ! n !} .
$$

In conclusion, finding a local optimum $\sigma^{*} \in S_{n}$ for the LOP under the swap neighborhood implies that $k_{n}^{S}$ solutions are not better than $\sigma^{*}$, where $k_{n}^{S}=\mathcal{M}_{n}$ is the $n$-th Motzkin Number. Moreover, we know how to build the only solutions that can be better than the local optimum: by applying a number of crossing swaps to the items of the local optimum. We can not guarantee the resultant solution to be better than the local optimum, but we know that by applying non-crossing swaps to $\sigma^{*}$ we do obtain solutions that will never be better than the local optimum.

\subsection{Analysis for the Insert}

Example. If $\sigma^{*}=(1234) \in S_{4}$ is a local optimum under the insert neighborhood, then $f\left(\sigma^{*}\right) \geq f(\sigma), \forall \sigma \in \mathcal{N}_{I}\left(\sigma^{*}\right)$. The inequalities obtained when comparing the objective function value of the local optimum $\sigma^{*}$ with the values of the neighbors are:

$$
\begin{aligned}
a_{12} & \geq a_{21} \\
a_{12}+a_{13} & \geq a_{21}+a_{31} \\
a_{12}+a_{13}+a_{14} & \geq a_{21}+a_{31}+a_{41} \\
a_{23} & \geq a_{32} \\
a_{23}+a_{24} & \geq a_{32}+a_{42} \\
a_{13}+a_{23} & \geq a_{31}+a_{32} \\
a_{34} & \geq a_{43} \\
a_{14}+a_{24}+a_{34} & \geq a_{41}+a_{42}+a_{43} \\
a_{24}+a_{34} & \geq a_{42}+a_{43}
\end{aligned}
$$

This result is generalized in Theorem 3 for any permutation size. 
Theorem 3. A solution $\sigma^{*}=\left(\sigma^{*}(1) \sigma^{*}(2) \cdots \sigma^{*}(n)\right) \in S_{n}$ is a local optimum for the LOP under the insert neighborhood if and only if the input matrix $A=\left[a_{i j}\right]_{n \times n}$ of the LOP instance fulfills:

$$
\begin{gathered}
\sum_{k=i+1}^{j} a_{\sigma^{*}(i) \sigma^{*}(k)} \geq \sum_{k=i+1}^{j} a_{\sigma^{*}(k) \sigma^{*}(i)}, \\
\forall i=1, \ldots, n-1, \forall j=i+1, \ldots, n, \\
\sum_{k=j}^{i-1} a_{\sigma^{*}(k) \sigma^{*}(i)} \geq \sum_{k=j}^{i-1} a_{\sigma^{*}(i) \sigma^{*}(k)}, \\
\forall i=3, \ldots, n, \forall j=1, \ldots, i-2 .
\end{gathered}
$$

Proof. Let us suppose that a permutation $\sigma^{*}=\left(\sigma^{*}(1) \sigma^{*}(2) \cdots \sigma^{*}(n)\right) \in S_{n}$ is a local optimum for the LOP under the insert neighborhood. We denote the neighbors of $\sigma^{*}$ by $\sigma_{i<j}$, where $\forall i=1 \ldots, n-1, \forall j=i+1, \ldots, n, \sigma_{i<j}=\left(\sigma^{*}(1) \cdots \sigma^{*}(i-1) \sigma^{*}(i+\right.$ 1) $\left.\cdots \sigma^{*}(j-1) \sigma^{*}(j) \sigma^{*}(i) \sigma^{*}(j+1) \cdots \sigma^{*}(n)\right)$ and $\sigma_{i>j}$, where $\forall i=3 \ldots, n, \forall j=1, \ldots, n-$ $2, \sigma_{i>j}=\left(\sigma^{*}(1) \cdots \sigma^{*}(j-1) \sigma^{*}(i) \sigma^{*}(j) \sigma^{*}(j+1) \cdots \sigma^{*}(i-1) \sigma^{*}(i+1) \cdots \sigma^{*}(n)\right)$. Thus, their objective function values are $\forall i=1, \ldots, n-1, \forall j=i+1, \ldots, n$ :

$$
f\left(\sigma_{i<j}\right)=f\left(\sigma^{*}\right)-\sum_{k=i+1}^{j} a_{\sigma^{*}(i) \sigma^{*}(k)}+\sum_{k=i+1}^{j} a_{\sigma^{*}(k) \sigma^{*}(i)}
$$

And $\forall i=3, \ldots, n, \forall j=1, \ldots, n-2$ :

$$
f\left(\sigma_{i>j}\right)=f\left(\sigma^{*}\right)-\sum_{k=j}^{i-1} a_{\sigma^{*}(k) \sigma^{*}(i)}+\sum_{k=j}^{i-1} a_{\sigma^{*}(i) \sigma^{*}(k)} .
$$

As $\sigma^{*}$ is a local optimum, $f\left(\sigma^{*}\right) \geq f\left(\sigma_{i<j}\right)$ and $f\left(\sigma^{*}\right) \geq f\left(\sigma_{i>j}\right), \forall \sigma_{i<j}, \sigma_{i>j} \in \mathcal{N}_{I}\left(\sigma^{*}\right)$ and therefore, the eq. (3) and (4) are fulfilled.

When comparing $f\left(\sigma^{*}\right)$ with $f\left(\sigma_{i<j}\right)$ and $f\left(\sigma_{i>j}\right), \forall \sigma^{*} \in S_{n}, \forall \sigma_{i<j}, \sigma_{i>j} \in \mathcal{N}_{I}\left(\sigma^{*}\right)$ :

$$
\begin{gathered}
f\left(\sigma_{i<j}\right)=f\left(\sigma^{*}\right)-\sum_{k=i+1}^{j} a_{\sigma^{*}(i) \sigma^{*}(k)}+\sum_{k=i+1}^{j} a_{\sigma^{*}(k) \sigma^{*}(i)} \\
f\left(\sigma_{i>j}\right)=f\left(\sigma^{*}\right)-\sum_{k=j}^{i-1} a_{\sigma^{*}(k) \sigma^{*}(i)}+\sum_{k=j}^{i-1} a_{\sigma^{*}(i) \sigma^{*}(k)} .
\end{gathered}
$$

If the inequalities in (3) and (4)are fulfilled for $\sigma^{*}$, then, $\forall i, j: f\left(\sigma_{i<j}\right) \leq f\left(\sigma^{*}\right)$ and $f\left(\sigma_{i>j}\right) \leq f\left(\sigma^{*}\right)$. So $\sigma^{*}$ is a local optimum. 
For this neighborhood, as in the previous two cases, there is a set of non-neighboring permutations that are also worse than or equal to the local optimum. This can be proved by considering a number of restrictions in (3) and (4). Let's consider again the permutation $\sigma^{*}=(123456) \in S_{6}$ as the local optimum (Figure 4 top-left). Inserting the item 1 of $\sigma^{*}$ into the 3rd position, the solution codified by the permutation $(231456) \in \mathcal{N}_{I}\left(\sigma^{*}\right)$ is obtained (Figure 4 top-right). When the objective function value of this solution is compared with that of the local optimum, the elements $a_{12}$ and $a_{13}$ (in red) are subtracted while $a_{21}$ and $a_{31}$ (in blue) are added. Thus, the sum of the elements in red must be greater than or equal to the sum of the elements in blue: $a_{12}+a_{13} \geq a_{21}+a_{31}$. The solution (123645) $\in \mathcal{N}_{I}\left(\sigma^{*}\right)$, obtained from inserting the item 6 of $\sigma^{*}$ into the 4 th position, is given in Figure 4 bottom-left. It is observed that the elements $a_{46}$ and $a_{56}$ (in red) are subtracted while $a_{64}$ and $a_{65}$ (in blue) are added when comparing with the objective function of $\sigma^{*}$, thus, $a_{46}+a_{56} \geq a_{64}+a_{65}$. Finally, the permutation $\sigma=(231645) \notin \mathcal{N}_{I}\left(\sigma^{*}\right)$ is the result of inserting the item 1 of $\sigma^{*}$ into the 3rd position and the item 6 of $\sigma^{*}$ into the 4 th position and represents the matrix given in Figure 4 bottom-right. It is observed that this solution fulfils that $f\left(\sigma^{*}\right) \geq f(\sigma)$.

\begin{tabular}{|c|c|c|c|c|c|c|c|c|c|c|c|c|c|}
\hline & & & & & & & & & & & & & \\
\hline & $a_{11}$ & $a_{12}$ & $a_{13}$ & $a_{14}$ & $a_{15}$ & $a_{16}$ & & $a_{22}$ & $a_{23}$ & $a_{21}$ & $a_{24}$ & $a_{25}$ & $a_{26}$ \\
\hline & $a_{21}$ & $a_{22}$ & $a_{23}$ & $a_{24}$ & $a_{25}$ & 26 & & $a_{32}$ & $a_{33}$ & $a_{31}$ & $a_{34}$ & $a_{35}$ & $l_{36}$ \\
\hline & $a_{31}$ & $a_{32}$ & $a_{33}$ & 34 & $a_{35}$ & 36 & & 12 & $a_{13}$ & 11 & $l_{14}$ & $a_{15}$ & $l_{1}$ \\
\hline & $a_{41}$ & $a_{42}$ & $a$ & & & 46 & & $l_{42}$ & $a_{43}$ & $a_{41}$ & 44 & $a_{45}$ & 4 \\
\hline & $a_{51}$ & $a_{52}$ & $a_{53}$ & $a_{54}$ & $a_{55}$ & $a_{56}$ & & $a_{52}$ & $a_{53}$ & $a_{51}$ & $a_{54}$ & $a_{55}$ & $l_{5}$ \\
\hline & $a_{61}$ & $a_{62}$ & $a_{63}$ & 64 & $a_{65}$ & 66 & & 62 & $a_{63}$ & $a_{61}$ & 64 & $a_{65}$ & \\
\hline & 1 & 2 & 3 & 6 & 4 & 5 & & 2 & 3 & 1 & 6 & 4 & 5 \\
\hline & $a_{11}$ & $a_{12}$ & c & 6 & 4 & 15 & & 22 & $a_{23}$ & $a_{21}$ & 26 & $a_{24}$ & $a_{2}$ \\
\hline & $a_{21}$ & $a_{22}$ & $a_{23}$ & $a_{26}$ & $a_{24}$ & $a_{25}$ & 3 & $a_{32}$ & $a_{33}$ & $a_{31}$ & $a_{36}$ & $a_{34}$ & $a_{35}$ \\
\hline & $a_{31}$ & 32 & $a_{33}$ & & $a_{34}$ & $a_{35}$ & & $a_{12}$ & $a_{13}$ & 11 & $l_{16}$ & 14 & $l_{15}$ \\
\hline & $a_{61}$ & 2 & a & $a_{66}$ & $a$ & 65 & 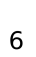 & $a_{62}$ & $a_{63}$ & $a_{61}$ & $a_{66}$ & $a_{64}$ & 65 \\
\hline & $a_{41}$ & $a_{42}$ & $a_{43}$ & $a_{46}$ & 44 & 45 & 4 & $l_{42}$ & $a_{43}$ & $a_{41}$ & $a_{46}$ & $a_{44}$ & $a_{45}$ \\
\hline & $a_{51}$ & $a_{52}$ & $a_{53}$ & $a_{56}$ & 54 & $a_{55}$ & 5 & $a_{52}$ & $a_{53}$ & $a_{51}$ & $a_{56}$ & $a_{54}$ & 55 \\
\hline
\end{tabular}

Figure 4: Matrix solutions of the LOP represented by the permutations (123456), (231456), (123645) and (231645). The elements that are involved in the objective function are shadowed, and comparing the objective function value of each solution with that of the local optimum, the elements subtracted are in red while the elements added are in blue.

However, we also find solutions which combine two or more insertion movements of items and for which we can not guarantee that they are not better than $\sigma^{*}$. For instance, taking $\sigma=(341256) \notin \mathcal{N}_{I}\left(\sigma^{*}\right)$, which is the result of inserting the item 3 into the first position and the item 4 into the second position of $\sigma^{*}$, we can not prove that 
$f\left(\sigma^{*}\right) \geq f(\sigma)$ just with restrictions in (3) and (4).

As it is observed, if the insertion movements do not cross with each other, then, one does not interfere in the objective function value of the other one. Thus, the solutions that are the result of applying at least two insertion movements to the items of the local optimum, in such a way that they do not cross with each other, will never be better than this local optimum. In fact, for $n=4$ there are only 3 permutations than can be better than a local optimum (it can easily be proved that the rest of permutations are the result of applying non-crossing intersection movements). In Example 3.3, these solutions are: (2413), (3142), (3412). In general, given a local optimum in $S_{4}, \sigma^{*}=\left(\sigma^{*}(1) \sigma^{*}(2) \sigma^{*}(3) \sigma^{*}(4)\right)$, just three solutions could be better than $\sigma^{*}:\left(\sigma^{*}(2) \sigma^{*}(4) \sigma^{*}(1) \sigma^{*}(3)\right),\left(\sigma^{*}(3) \sigma^{*}(1) \sigma^{*}(4) \sigma^{*}(2)\right)$ and $\left(\sigma^{*}(3) \sigma^{*}(4) \sigma^{*}(1) \sigma^{*}(2)\right)$. So as to extrapolate this aspect to the general case $n$, in Theorem 4 the necessary condition for any permutation of size $n$ to be better than a local optimum under the insert neighborhood is formulated.

Theorem 4. Given a local optimum $\sigma^{*}=\left(\sigma^{*}(1) \ldots \sigma^{*}(n)\right) \in S_{n}$ of the LOP under the insert neighborhood, then any permutation $\sigma \in S_{n}$ that contains a subsequence which maintains the same relative order between the items with respect to $\sigma^{*}$ as in

$$
\begin{aligned}
& \left(\sigma^{*}(2) \sigma^{*}(4) \sigma^{*}(1) \sigma^{*}(3)\right), \\
& \left(\sigma^{*}(3) \sigma^{*}(1) \sigma^{*}(4) \sigma^{*}(2)\right), \\
& \left(\sigma^{*}(3) \sigma^{*}(4) \sigma^{*}(1) \sigma^{*}(2)\right),
\end{aligned}
$$

could be better than $\sigma^{*}$.

Note: the order is determined by $\sigma^{*}: \forall i \neq j, \sigma^{*}(i)<\sigma^{*}(j)$ iff $i<j$.

Proof. A permutation $\sigma \in S_{n}$ contains a subsequence of items of the form (2413) if $\exists 1 \leq d<e<g<h \leq n$ such that $\sigma(d)=2, \sigma(e)=4, \sigma(g)=1, \sigma(h)=3$. For example, $\sigma=(75264183)$, contains the subsequence (2413) because $\sigma(3)=2, \sigma(5)=$ $4, \sigma(6)=1, \sigma(8)=3$. A permutation $\sigma \in S_{n}$ contains a subsequence which maintains the same relative order between the items as in (2413) if $\exists 1 \leq d<e<g<h \leq n$ such that $\sigma(g)<\sigma(d)<\sigma(h)<\sigma(e)$. The order is related to $\sigma^{*}$. This means that, for example, if $\sigma^{*}=(246351)$, the solution $\sigma=(361452)$ contains the subsequence $\left(\sigma^{*}(4) \sigma^{*}(6) \sigma^{*}(2) \sigma^{*}(5)\right)=(3145)$ with the same relative order with respect to $\sigma^{*}$ as $\left(\sigma^{*}(2) \sigma^{*}(4) \sigma^{*}(1) \sigma^{*}(3)\right)=(4326)$.

We start showing what happens with the solutions containing the three mentioned subsequences, and then, we will do a generalization for the cases with the same relative order. The objective function value of $\sigma^{*}$ is: $f\left(\sigma^{*}\right)=\sum_{i=1}^{n-1} \sum_{j=i+1}^{n} a_{\sigma^{*}(i) \sigma^{*}(j)}$.

- If $\sigma=\left(\cdots \sigma^{*}(2) \cdots \sigma^{*}(4) \cdots \sigma^{*}(1) \cdots \sigma^{*}(3) \cdots\right)$ then

$$
\begin{gathered}
f(\sigma)=\ldots+a_{\sigma^{*}(2) \sigma^{*}(4)}+\ldots+a_{\sigma^{*}(2) \sigma^{*}(1)}+\ldots+a_{\sigma^{*}(2) \sigma^{*}(3)}+\ldots+ \\
+\ldots+a_{\sigma^{*}(4) \sigma^{*}(1)}+\ldots+a_{\sigma^{*}(4) \sigma^{*}(3)}+\ldots+a_{\sigma^{*}(1) \sigma^{*}(3)}+\ldots
\end{gathered}
$$

Comparing with $f\left(\sigma^{*}\right): f\left(\sigma^{*}\right)-f(\sigma)=$

$$
\begin{gathered}
=\left(a_{\sigma^{*}(1) \sigma^{*}(2)}+a_{\sigma^{*}(1) \sigma^{*}(3)}+a_{\sigma^{*}(1) \sigma^{*}(4)}+\ldots+\right. \\
\left.+a_{\sigma^{*}(2) \sigma^{*}(3)}+a_{\sigma^{*}(2) \sigma^{*}(4)}+\ldots+a_{\sigma^{*}(3) \sigma^{*}(4)}+\ldots\right)-
\end{gathered}
$$




$$
\begin{gathered}
-\left(\ldots+a_{\sigma^{*}(2) \sigma^{*}(4)}+\ldots+a_{\sigma^{*}(2) \sigma^{*}(1)}+\ldots+a_{\sigma^{*}(2) \sigma^{*}(3)}+\right. \\
\left.+\ldots+a_{\sigma^{*}(4) \sigma^{*}(1)}+\ldots+a_{\sigma^{*}(4) \sigma^{*}(3)}+\ldots+a_{\sigma^{*}(1) \sigma^{*}(3)}+\ldots\right)= \\
\quad=\left(a_{\sigma^{*}(1) \sigma^{*}(2)}+a_{\sigma^{*}(1) \sigma^{*}(4)}+\ldots+a_{\sigma^{*}(3) \sigma^{*}(4)}+\ldots\right)- \\
-\left(\ldots a_{\sigma^{*}(2) \sigma^{*}(1)}+\ldots+a_{\sigma^{*}(4) \sigma^{*}(1)}+\ldots+a_{\sigma^{*}(4) \sigma^{*}(3)}+\ldots\right) .
\end{gathered}
$$

Looking at the equations in (3) and (4), we observe that $a_{\sigma^{*}(1) \sigma^{*}(4)}$ appears in the following cases: (i) when both $a_{\sigma^{*}(1) \sigma^{*}(2)}$ and $a_{\sigma^{*}(1) \sigma^{*}(3)}$ appear, and (ii) when both $a_{\sigma^{*}(2) \sigma^{*}(4)}$ and $a_{\sigma^{*}(3) \sigma^{*}(4)}$ appear. As $a_{\sigma^{*}(1) \sigma^{*}(3)}$ and $a_{\sigma^{*}(2) \sigma^{*}(4)}$ are common factors in both $f\left(\sigma^{*}\right)$ and $f(\sigma)$, then they do not appear in the difference between these functions; (i) and (ii) are not fulfilled. So, we can not prove that $f\left(\sigma^{*}\right) \geq f(\sigma)$.

- If $\sigma=\left(\cdots \sigma^{*}(3) \cdots \sigma^{*}(1) \cdots \sigma^{*}(4) \cdots \sigma^{*}(2) \cdots\right)$ then $f(\sigma)=\sum_{i=1}^{n-1} \sum_{j=i+1}^{n} a_{\sigma(i) \sigma(j)}$. Comparing with $f\left(\sigma^{*}\right)$ :

$$
\begin{gathered}
f\left(\sigma^{*}\right)-f(\sigma)=\left(a_{\sigma^{*}(1) \sigma^{*}(3)}+a_{\sigma^{*}(2) \sigma^{*}(3)}+\ldots+a_{\sigma^{*}(2) \sigma^{*}(4)}+\ldots\right)- \\
-\left(\ldots+a_{\sigma^{*}(3) \sigma^{*}(1)}+\ldots+a_{\sigma^{*}(3) \sigma^{*}(2)}+a_{\sigma^{*}(4) \sigma^{*}(2)}+\ldots\right) .
\end{gathered}
$$

Looking at equations in (3) and (4), we observe that $a_{\sigma^{*}(1) \sigma^{*}(3)}$ appears in the following cases: (i) when $a_{\sigma^{*}(1) \sigma^{*}(2)}$ appears and (ii) when $a_{\sigma^{*}(2) \sigma^{*}(3)}$ appears. While $a_{\sigma^{*}(2) \sigma^{*}(4)}$ appears in the following cases: (iii) when $a_{\sigma^{*}(2) \sigma^{*}(3)}$ appears and (iv) when $a_{\sigma^{*}(3) \sigma^{*}(4)}$ appears. As $a_{\sigma^{*}(1) \sigma^{*}(2)}$ and $a_{\sigma^{*}(3) \sigma^{*}(4)}$ are common factors in both $f\left(\sigma^{*}\right)$ and $f(\sigma)$, then they do not appear in the difference between these functions. The factor $a_{\sigma^{*}(2) \sigma^{*}(3)}$ does appear, but just once. So, although (ii) can be fulfilled, (iv) can not (or vice versa). Thus, we can not prove that $f\left(\sigma^{*}\right) \geq f(\sigma)$.

- The case $\sigma=\left(\cdots \sigma^{*}(3) \cdots \sigma^{*}(4) \cdots \sigma^{*}(1) \cdots \sigma^{*}(2) \cdots\right)$ is proved in the same way as the previous two cases.

Let us generalize these three cases to those permutations that do not necessarily contain these subsequences, but they do contain a subsequence that maintains the same relative order.

- If $\sigma=\left(\cdots \sigma^{*}(e) \cdots \sigma^{*}(h) \cdots \sigma^{*}(d) \cdots \sigma^{*}(g) \cdots\right)$ with $1 \leq d<e<g<h \leq n$, then

$$
\begin{aligned}
& f(\sigma)=\ldots+a_{\sigma^{*}(e) \sigma^{*}(h)}+\ldots+a_{\sigma^{*}(e) \sigma^{*}(d)}+\ldots+a_{\sigma^{*}(e) \sigma^{*}(g)}+ \\
& +\ldots+a_{\sigma^{*}(h) \sigma^{*}(d)}+\ldots+a_{\sigma^{*}(h) \sigma^{*}(g)}+\ldots+a_{\sigma^{*}(d) \sigma^{*}(g)}+\ldots
\end{aligned}
$$

Comparing with $f\left(\sigma^{*}\right): f\left(\sigma^{*}\right)-f(\sigma)=$

$$
\begin{aligned}
= & \left(\ldots+a_{\sigma^{*}(d) \sigma^{*}(e)}+\ldots+a_{\sigma^{*}(d) \sigma^{*}(h)}+\ldots+a_{\sigma^{*}(g) \sigma^{*}(h)}+\ldots\right)- \\
& -\left(\ldots+a_{\sigma^{*}(e) \sigma^{*}(d)}+\ldots+a_{\sigma^{*}(h) \sigma^{*}(d)}+\ldots+a_{\sigma^{*}(h) \sigma^{*}(g)}+\ldots\right)
\end{aligned}
$$

Looking at the equations in (3) and (4), we observe that $a_{\sigma^{*}(d) \sigma^{*}(h)}$ with $d<h$ and $d$ and $h$ non-consecutive numbers appears in the following cases: (i) when $a_{\sigma^{*}(d) \sigma^{*}(d+1)}, a_{\sigma^{*}(d) \sigma^{*}(d+2)}, \ldots, a_{\sigma^{*}(d) \sigma^{*}(h-1)}$ appear, and (ii) when $a_{\sigma^{*}(h-1) \sigma^{*}(h)}$, $a_{\sigma^{*}(h-2) \sigma^{*}(h)}, \ldots, a_{\sigma^{*}(d+1) \sigma^{*}(h)}$ appear. As $a_{\sigma^{*}(d) \sigma^{*}(g)}$ (with $d<g<h$ ) and $a_{\sigma^{*}(e) \sigma^{*}(h)}$ (with $h>e>d$ ) are common factors in both $f\left(\sigma^{*}\right)$ and $f(\sigma)$, then they do not appear in the difference between these functions; (i) and (ii) are not fulfilled. So, we can not prove that $f\left(\sigma^{*}\right) \geq f(\sigma)$. 
- The cases $\sigma=\left(\cdots \sigma^{*}(g) \cdots \sigma^{*}(d) \cdots \sigma^{*}(h) \cdots \sigma^{*}(e) \cdots\right)$ and $\sigma=$ $\left(\cdots \sigma^{*}(g) \cdots \sigma^{*}(h) \cdots \sigma^{*}(d) \cdots \sigma^{*}(e) \cdots\right)$ with $1 \leq d<e<g<h \leq n$ are proved in the same way as the previous case.

Corollary 1. Given a local optimum $\sigma^{*}=\left(\sigma^{*}(1) \ldots \sigma^{*}(n)\right) \in S_{n}$ of the LOP under the insert neighborhood, if a permutation $\sigma \in S_{n}$ does not contain any subsequence which maintains the same relative order between the items with respect to $\sigma^{*}$ as in

$$
\begin{aligned}
& \left(\sigma^{*}(2) \sigma^{*}(4) \sigma^{*}(1) \sigma^{*}(3)\right), \\
& \left(\sigma^{*}(3) \sigma^{*}(1) \sigma^{*}(4) \sigma^{*}(2)\right), \\
& \left(\sigma^{*}(3) \sigma^{*}(4) \sigma^{*}(1) \sigma^{*}(2)\right),
\end{aligned}
$$

then $\sigma$ is necessarily worse than $\sigma^{*}$.

Proof. The proof is similar to that of Theorem 4. Just considering the rest of subsequences, it can easily be proved that in all of the cases, a number of equations in (3) and (4) are fulfilled.

In practice, one could inmediately build a number of permutations of size $n$ that fulfil Theorem 4 . Nevertheless, the generalization about the exact number of this kind of permutations for any $n$ is not an immediate issue. We may refer to the problem known in the literature as Permutation Pattern Avoidance (Linton et al., 2010). It consists of obtaining the number of all the permutations of size $n$ avoiding some patterns. It was proved that the number of permutations of size $n$ avoiding $K$ patterns does not need to be the same as the number of permutations of the same size $n$ avoiding $K$ distinct patterns. In our case, we would be interested in the number of permutations of size $n$ avoiding the three patterns (2413), (3142) and (3412), because Permutation Pattern Avoidance is equivalent for different permutations that maintain the same relative order. Unfortunately, this is an unsolved problem. However, on the one hand, the number of permutations of size $n$ avoiding the patterns (2413), (3142), (3412) and (2143) is already solved (Waton, 2007). So a lower bound for our problem is known. On the other hand, the number of permutations of size $n$ avoiding the patterns (2413) and (3142) is also solved (Avis and Newborn, 1981) and they are given by the Large Schröder Numbers (Rogers, 1977; Schroder, 1870). Thus, an upper bound for our problem is also known.

The expression for the number of permutations of size $n$ avoiding the patterns (2413), (3142), (3412) and (2143) is:

$$
w_{n}=4 w_{n-1}-2 w_{n-2}, \forall n \geq 3
$$

with $w_{1}=1$ and $w_{2}=2$. It is a lower bound for $k_{n}^{I}$, the number of permutations that are necessarily worse than or equal to a given local optimum under the insert neighborhood.

The Large Schröder Numbers of size $n$ are defined by the following recurrence relation:

$$
\mathcal{S}_{n}=3 \mathcal{S}_{n-1}+\sum_{i=1}^{n-3} \mathcal{S}_{i} \mathcal{S}_{n-i-1}, \forall n \geq 3
$$


Table 1: Number of neighboring solutions, including the local optimum and number of solutions, that are necessarily worse than or equal to a given local optimum for different permutation sizes according to the neighborhood considered.

\begin{tabular}{lcrrrrrrrrrrrr}
\hline \multicolumn{2}{c}{ Permutation size $n$} & 2 & 3 & 4 & 5 & 6 & 7 & 8 & 10 & 12 & 14 & 18 & 20 \\
\hline \multirow{2}{*}{ Adjacent } & $(n-1)+1$ & 2 & 3 & 4 & 5 & 6 & 7 & 8 & 10 & 12 & 14 & 18 & 20 \\
& $k_{n}^{A}$ & 2 & 3 & 5 & 8 & 13 & 21 & 34 & 89 & 233 & 610 & 4181 & 10946 \\
\hline \multirow{2}{*}{ Swap } & $n(n-1) / 2+1$ & 2 & 4 & 7 & 11 & 16 & 22 & 29 & 46 & 67 & 92 & 154 & 5082 \\
& $k_{n}^{S}$ & 2 & 4 & 9 & 21 & 51 & 127 & 323 & 2188 & 15511 & 113634 & 6536382 & 50852019 \\
\hline \multirow{4}{*}{ Insert } & $(n-1)^{2}+1$ & 2 & 5 & 10 & 17 & 26 & 37 & 50 & 82 & 122 & 170 & 290 & 362 \\
& $k_{n}^{I} \geq$ & 2 & 6 & 20 & 68 & 232 & 792 & 2704 & 31520 & 367424 & 4283008 & 581984768 & 6784111616 \\
& $k_{n}^{I} \leq$ & 2 & 6 & 22 & 90 & 394 & 1806 & 8558 & 206098 & 5293446 & 142078746 & 111818026018 & 3236724317174 \\
\hline
\end{tabular}

with $\mathcal{S}_{1}=1$ and $\mathcal{S}_{2}=2$. It is an upper bound for $k_{n}^{I}$.

In conclusion, finding a local optimum $\sigma^{*} \in S_{n}$ for the LOP under the insert neighborhood implies that the number of permutations avoiding the patterns (2413), (3142), and (3412) is the number of solutions that will never be better than $\sigma^{*}$. Moreover, we know how to build the solutions that can be better than the local optimum: by considering those solutions containing subsequences that maintain the relative order of $\left(\sigma^{*}(2) \sigma^{*}(4) \sigma^{*}(1) \sigma^{*}(3)\right),\left(\sigma^{*}(3) \sigma^{*}(1) \sigma^{*}(4) \sigma^{*}(2)\right)$ and/or $\left(\sigma^{*}(3) \sigma^{*}(4) \sigma^{*}(1) \sigma^{*}(2)\right)$.

\section{Relevance of the new insights}

Considering the three neighborhoods, adjacent swap, swap and insert, we have explored the different landscapes generated for the LOP. We focus on the number of solutions that one could discard from the search space when a local optimum is known, because they will not be better than the local optimum. In Table 1, the number of those solutions is shown for the adjacent swap $\left(k_{n}^{A}\right)$ and the swap $\left(k_{n}^{S}\right)$ neighborhoods, for different permutation sizes. For the insert neighborhood, as we do not know the exact number $k_{n}^{I}$, the lower and the upper bounds are indicated. As the three cases $k_{n}^{A}, k_{n}^{S}$ and $k_{n}^{I}$ include the local optimum, we compare them with the sizes of the neighborhoods adding the local optimum: $\left|\mathcal{N}_{A}\right|+1,\left|\mathcal{N}_{S}\right|+1$ and $\left|\mathcal{N}_{I}\right|+1$, respectively.

As can be observed, as $n$ grows, in the three neighborhoods there is a significant difference between both values. Of course, the one with the highest difference is the insert neighborhood. Just as a reference, for $n=20$, when a local optimum under the insert neighborhood is found, not only the 362 neighbors can be discarded as worse solutions, but an amount between $6.8 \times 10^{9}$ and $3.2 \times 10^{12}$ could be avoided. The most interesting result is that we do not only know the number of solutions that are worse, but we do know which these solutions are and how we can build a new solution in order to not fall into the set of these permutations that we already know are worse. In fact, in both swap and insert neighborhoods, it was already shown that the local optima are located inside the first half of solutions. That is, understanding an instance of the LOP as a ranking of the solutions from better to worse (Hernando et al., 2019b) we could say that the local optima belong to the upper half of the ranking (they are better than their reverse permutation). In this sense, one could conclude that there are at least $n ! / 2$ solutions worse than a local optimum. This statement is true; nevertheless, no 
evidence about which solutions these are exactly and how we can avoid them has been given. So, this is the main difference with our work, in which we know how to discard a huge number of solutions. Of course, the information drawn from Section 3 can be immediately applied to any algorithm designed to solve LOP instances; particularly, for efficient strategies to escape from the local optima in iterated local search or variable neighborhood search techniques.

\section{Conclusions and Future Work}

In this paper, three landscapes for the linear ordering problem have been analyzed. This work focuses on the fact that knowledge can be collected once a local optimum is found under a specific neighborhood basing on the $\Delta$-evaluation functions. In this sense, as by definition a local optimum is not worse than its neighbors, the input matrix of the problem instance must fulfill a number of restrictions. These restrictions are valuable information about the landscape, because a combination of them can be used to prove that a number of solutions, apart from the neighbors of the local optimum, are not better than the local optimum.

For the three neighborhoods analyzed, the number of these solutions that are necessarily worse than the local optimum $\left(k_{n}\right)$ is connected to concepts found in the Combinatorics field. To the best of our knowledge, this connection was never made before. In the case of the adjacent swap neighborhood, $k_{n}^{A}$ is the $(n+1)$-th Fibonacci number. For the swap neighborhood, we have found that the number $k_{n}^{S}$ is the $n$-th Motzkin number. In the insert neighborhood case, we have observed that $k_{n}^{I}$ is the number of permutations of size $n$ which avoid the patterns (2413), (3142), (3412). In the Combinatorics arena, this problem is known as the Permutation Pattern Avoidance. Unfortunately, our specific case is an unsolved problem in the literature. However, we can find a lower bound and an upper bound. The upper bound is related with the Schröder numbers. This number $k_{n}$ is considerably larger than the number of neighbors: while for $n=20$, for the adjacent swap, swap and insert, there are just $19,190,361$ neighbors of a local optimum, respectively, the number of solutions that are necessarily worse than or equal to the local optimum are $10946,5.08 \times 10^{7}$ and between $6.78 \times 10^{9}$ and $3.24 \times 10^{12}$, respectively. The most interesting aspect of the new findings presented in this paper, is that we know how to build those solutions that are not inside this set of non-better solutions. In the three cases, one just need to apply a number of movements of the corresponding neighborhood in such a way that one item is involved in more than one movement.

The theoretical analysis provided is valuable knowledge for those algorithms that are designed to escape from the local optima. This information will help to improve the efficiency of the existing algorithms or to develop new intelligent techniques. In fact, we plan to design a strategy to escape from the local optima taking into account the information drawn from this paper.

\section{Acknowledgements}

This work has been partially supported by the Spanish Ministry of Economy, Industry and Competitiveness (TIN2016-78365-R), and the Basque Government (IT1244-19 and ELKARTEK programs). Jose A. Lozano is also supported by BERC 2018-2021 (Basque government) and BCAM Severo Ochoa accreditation SEV-2017-0718 (Spanish Ministry 
of Science and Innovation).

\section{References}

M. Aigner. 1998. Motzkin Numbers. European Journal of Combinatorics 19, 6 (1998), 663 -675 .

A.A. Albrecht, P.C.R. Lane, and K. Steinhofel. 2008. Combinatorial landscape analysis for k-SAT instances. In Evolutionary Computation, 2008. CEC 2008. (IEEE World Congress on Computational Intelligence). IEEE Congress on. 2498 -2504.

Khulood Alyahya and Jonathan E. Rowe. 2014. Evolutionary Computation in Combinatorial Optimisation: 14th European Conference, EvoCOP 2014, Granada, Spain, April 23-25, 2014, Revised Selected Papers. Springer Berlin Heidelberg, Berlin, Heidelberg, Chapter Phase Transition and Landscape Properties of the Number Partitioning Problem, 206-217.

D. Avis and M. Newborn. 1981. On pop-stacks in series. Utilitas Math. 19 (1981), 129-140.

Josu Ceberio, Alexander Mendiburu, and Jose A. Lozano. 2015. The linear ordering problem revisited. European Journal of Operational Research 241, 3 (2015), 686 - 696.

F. Chicano, F. Daolio, G. Ochoa, S. Verel, M. Tomassini, and E. Alba. 2012. Local optima networks, landscape autocorrelation and heuristic search performance. In Proceedings of Parallel Problem Solving from Nature, PPSN XII (Lecture Notes in Computer Science), Vol. 7492. Springer, 337-347.

C. G Garcia, D. Perez-Brito, V. Campos, and R. Marti. 2006. Variable neighborhood search for the linear ordering problem. Computers and operations research 33, 12 (2006), 3549-3565.

Pierre Hansen and Nenad Mladenović. 2018. Variable Neighborhood Search. Springer International Publishing, Cham, 759-787.

Leticia Hernando, Alexander Mendiburu, and Jose A. Lozano. 2018. Hill-climbing algorithm: let's go for a walk before finding the optimum. In Proceeding of 2018 IEEE Congress on Evolutionary Computation. 1292-1298.

Leticia Hernando, Alexander Mendiburu, and Jose A. Lozano. 2019a. Anatomy of the Attraction Basins: Breaking with the Intuition. Evolutionary Computation 27, 3 (2019), 435-466.

Leticia Hernando, Alexander Mendiburu, and Jose A. Lozano. 2019b. Characterising the Rankings Produced by Combinatorial Optimisation Problems and Finding their Intersections. In In Proceedings of The Genetic and Evolutionary Computation Conference. 266-273.

Jérémie Humeau, Arnaud Liefooghe, El-Ghazali Talbi, and Sébastien Verel. 2013. ParadisEO-MO: From Fitness Landscape Analysis to Efficient Local Search Algorithms. Journal of Heuristics 19, 6 (2013), 881-915.

Steve Linton, Nik Ruškuc, and Vincent Vatter. 2010. Permutation Patterns. Vol. 376. Cambridge University Press. 
Helena R. Lourenço, Olivier C. Martin, and Thomas Stützle. 2003. Handbook of Metaheuristics. Springer US, Boston, MA, Chapter Iterated Local Search, 320-353.

Helena R. Lourenço, Olivier C. Martin, and Thomas Stützle. 2010. Handbook of Metaheuristics. Springer US, Boston, MA, Chapter Iterated Local Search: Framework and Applications, 363-397.

Marie-Eléonore Marmion, Clarisse Dhaenens, Laetitia Jourdan, Arnaud Liefooghe, and Sébastien Verel. 2011. NILS: A Neutrality-Based Iterated Local Search and Its Application to Flowshop Scheduling. In Evolutionary Computation in Combinatorial Optimization: 11th European Conference, EvoCOP 2011, Peter Merz and Jin-Kao Hao (Eds.). Springer Berlin Heidelberg, Torino, Italy, 191-202.

Rafael Marti and Gerhard Reinelt. 2011. The Linear Ordering Problem: Exact and Heuristic Methods in Combinatorial Optimization. Springer-Verlag Berlin Heidelberg.

Sounaka Mishra and Kripasindhu Sikdar. 2004. On approximability of linear ordering and related NP-optimization problems on graphs. Discrete Applied Mathematics 136, 2-3 (2004), 249-269.

N. Mladenović and P. Hansen. 1997. Variable neighborhood search. Computers $\mathcal{E}$ Operations Research 24, 11 (1997), 1097 - 1100.

C. M. Reidys and P. F. Stadler. 2002. Combinatorial Landscapes. SIAM Rev. 44, 1 (2002), 3-54.

John Riordan. 1968. Combinatorial Identities. Krieger Publishing Company.

D. G. Rogers. 1977. A Schroder Triangle. In Combinatorial Mathematics V: Proceedings of the Fifth Australian Conference, Springer-Verlag (Ed.). 175-196.

Valentino Santucci and Josu Ceberio. 2020. Using pairwise precedences for solving the linear ordering problem. Applied Soft Computing 87 (2020), 1-15.

Tommaso Schiavinotto and Thomas Stützle. 2003. Search space analysis of the linear ordering problem. In Workshops on Applications of Evolutionary Computation. 322-333.

Tommaso Schiavinotto and Thomas Stützle. 2005. The Linear Ordering Problem: Instances, Search space analysis and algorithms. Journal of Mathematical Modelling and Algorithms 3, 4 (2005), 367-402.

E. Schroder. 1870. Vier kombinatorische Probleme. Z. Math. Phys. 15, 361-376 (1870).

T.C. Scott and P. Marketos. 2014. On the Origin of the Fibonacci Sequence. In MacTutor History of Mathematics archive. University of St Andrews.

Thomas Stützle. 2006. Iterated local search for the quadratic assignment problem. European Journal of Operational Research 174, 3 (2006), 1519 - 1539.

S. Waton. 2007. On permutation classes defined by token passing networks, gridding matrices and pictures: three flavours of involvement. Ph.D. Dissertation. University of St. Andrews. 